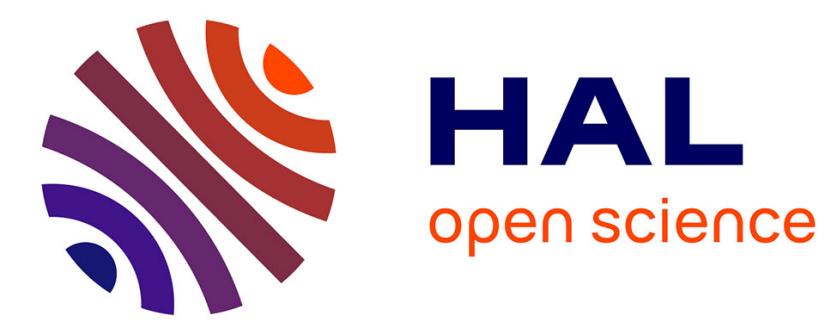

\title{
Un phare, une flotte, Isis, Faustine et l'annone Laurent Bricault
}

\section{To cite this version:}

Laurent Bricault. Un phare, une flotte, Isis, Faustine et l'annone. Chronique d'Egypte; bulletin periodique de la Fondation egyptologique reine Elisabeth, 2000, 75 (150), pp.136 - 149. hal-01817075

\section{HAL Id: hal-01817075 \\ https://hal.science/hal-01817075}

Submitted on 22 Jun 2018

HAL is a multi-disciplinary open access archive for the deposit and dissemination of scientific research documents, whether they are published or not. The documents may come from teaching and research institutions in France or abroad, or from public or private research centers.
L'archive ouverte pluridisciplinaire HAL, est destinée au dépôt et à la diffusion de documents scientifiques de niveau recherche, publiés ou non, émanant des établissements d'enseignement et de recherche français ou étrangers, des laboratoires publics ou privés. 


\section{CHR O N I U E D'É G Y P T E}

\section{LXXV (2000)}

Fasc. 149

EXTRAIT

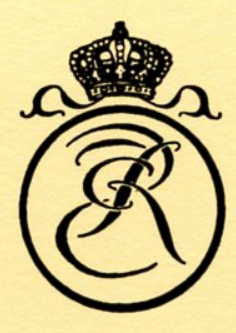

FONDATION ÉGYPTOLOGIQUE REINE ÉLISABETH EGYPTOLOGISCHE STICHTING KONINGIN ELISABETH 


\section{Un phare, une flotte, Isis, Faustine et l'annone}

Une inscription alexandrine, publiée en premier lieu par J.-Y. Empereur ${ }^{1}$, puis republiée par A. et E. Bernand $\left(^{2}\right)$, offre le premier exemple connu de l'attribution à Faustine la Jeune, épouse de Marc Aurèle, des épiclèses papía et $\sigma \omega \sigma i \sigma \tau o \lambda \mathrm{o}$. L'inscription émane des membres d'une association en charge des effigies de Marc Aurèle, Lucius Verus et Faustine Pharia, Sôsistolos, Nouvelle Auguste en l'honneur d'un certain P. Aelius Panopaios ( $\left.{ }^{3}\right)$, leur confrère, ancien procurateur impérial. Le texte est à dater entre 147 et 169 après J.-C.

Ce document nous offre l'occasion de reprendre le dossier de l'épiclèse Фapía / Pharia, et de tenter d'en préciser le sens, sinon les sens, et de définir le ou les rôles dévolus à celles qui la portent: Isis et désormais, également, Faustine la Jeune.

Dans la littérature moderne et savante, l'épiclèse Pharia est employée généralement comme synonyme de Pelagia ou d'Euploia, sans qu'une nette distinction apparaisse entre les trois, ni qu'une attitude rigoureuse soit perceptible dans le choix de tel ou tel qualificatif, ce malgré les prudentes réserves émises par $\mathrm{Ph}$. Bruneau au fil de trois articles dont la fine pertinence séduit toujours $\left({ }^{4}\right)$. Non seulement cet auteur invitait à ne pas confondre les trois épithètes, mais il soulignait la difficulté de rapporter un type précis de représentation figurée à l'une ou l'autre d'entre elles. Sur ces différents points, la prudence demeure et demeurera tant qu'une étude poussée des attributions marines et maritimes d'Isis — «Notre-Dame-des-flots» $\left.{ }^{5}\right)$ — n'aura pas été menée à bien $\left(^{6}\right)$

(1) Publication partielle de l'inscription, avec une photographie, dans Le Monde Copte 27-28 (1997), p. 31 (signalée dans le $B C H 118,1994$, p. 511 et $S E G$ XLIV 1442).

(2) A et E BERNAND, «Un procurateur des effigies impériales à Alexandrie», ZPE 122 (1998), pp. 97-101.

(3) Il s' (3) fils d'un Alexandrin ayant obtenu la citoyenneté romaine d'Hadrien.

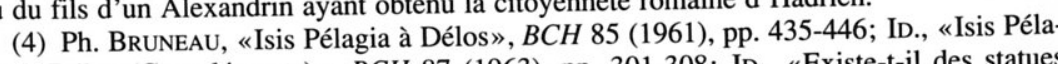
gia à Délos (Compléments)», $B C H 87$ (1963), pp. 301-308; ID., «Existe-t-il des statue d'Isis Pélagia?», BCH 98 (1974), pp. 333-381.

5) Pour reprendre la belle expression choisie par R. Turcan comme sous-titre des pages consacrées aux divinités égyptiennes dans ses Cultes orientaux dans le monde romai (Paris, 1989), p. 77.

(6) Voir l'Annexe ci-après.
L'épiclèse Pharia est attestée pour Isis dans les sources littéraires, épigraphiques et numismatiques ${ }^{7}$ ). Elle apparaît dans les premières à la fin du $\mathrm{I}^{\text {er }}$ s. avant J.-C., dans les deuxièmes au $\mathrm{II}^{\mathrm{e}}$ s. après J.-C., et dans les troisièmes au IV ${ }^{\mathrm{e}} \mathrm{s}$. après $\mathrm{J}$.-C.

Tibulle, le premier, évoque la «foule des célébrants de Pharos» $\left({ }^{8}\right)$, tandis qu'Ovide $\left({ }^{9}\right)$, avec à sa suite Martial $\left({ }^{10}\right)$ et Stace $\left({ }^{11}\right)$, parlent de la iuvenca Pharia, la génisse de Pharos, qui n'est autre qu'Isis-Io $\left({ }^{12}\right)$. Le même Stace $\left({ }^{13}\right)$, à propos d'Io parvenue sur le sol d'Égypte, écrit que «maintenant, [elle est] reine du Pharos». Ce toponyme n'est qu'un substitut du mot «Égypte», comme le prouvent ses autres occurrences dans l'œuvre du poète ${ }^{\left({ }^{14}\right)}$. Chez Ovide, le Pharos désigne clairement par métonymie Alexandrie $\left({ }^{15}\right)$, ce qui doit être également le cas dans le vers de Tibulle cité plus haut, ainsi que chez Apulée $\left({ }^{16}\right)$. Force est de constater que si ambiguïté il y a, elle ne porte que sur le fait de savoir si Pharos, chez les Latins, désigne seulement Alexandrie ou bien l'Égypte entière. Le même doute subsiste à propos de la citation qu'Eusèbe de Césarée fait de Porphyre, lorsque celui-ci évoque, parmi d'autres divinités, Isis de Pharos $\left({ }^{17}\right)$, comme pour celle, en discours indirect rapporté qui plus est, que Minucius Felix ferait d'Évhémère ${ }^{\left({ }^{18}\right)}$. Il en va probablement de même

(7) Cf. L. Bricault, Myrionymi. Les épiclèses grecques et latines d'Isis, de Sarapis et d'Anubis, (Stuttgart - Leipzig, 1996), pp. 72 et 89. La plupart des références littéraires et épigraphiques déjà chez A. CALDERINI, Dizionario. I (1935), p. 161; voir également les notes de P.M. FraSer, Ptolemaic Alexandria (Oxford, 1972), I pp. 20-21 et II, pp. 54-56, nn. 125-128. Je ne connais pas d'occurrence papyrologique de cette épithète.

(8) I, 3, 32 (trad. M. Ponchont, CUF).

(9) Ars amat. III, 635 et Fastes V, 619. Cf. également Pont. I, 1, 38

(10) Ep. X, 48.

(11) Théb. I, 254

(12) Il en est de même pour l'occurrence chez EusÈBE, Chronica, apud THÉOdORE LE SyNCELLE 237,6-9 (Schoene).

(13) Silv. III, II, 102.

(14) Silv. III, II, 112 et V, III, 244.

(15) Dans $A m$. II, 13,9, Ovide rappelle qu'Isis possède des sanctuaires à Parétonium, Canope, Memphis et Pharos. L'absence d'Alexandrie dans ce vers indique clairement que la cité se cache par métonymie sous le nom de son monument le plus emblématique, au moins pour un Latin; même figure de style dans Métam. IX, 773-774. Voir également les remarques d'A. RamireZ DE Verger, «The Text of Ovid, Amores 2.13.17-18», AJPh 109 (1988), pp. 87-89.

16) Métam. II, 28. 309F Smith p. 358) apud EusEBe, Praep. Ev. V, 7, 5.

(18) Octavius XXI, 1. Isis, humaine divinisée, est ici mise sur le même plan que Jupiter de Dicté ou Apollon de Delphes. 
pour les deux dernières attestations littéraires de l'épiclèse, chez Tertullien $\left({ }^{(9)}\right)$ et dans le Carmen in paganos $\left.{ }^{(20}\right)$. Aucun de ces textes ne justifie en lui-même la remarque fréquente selon laquelle le Pharos serait un centre important du culte d'Isis, où la déesse aurait eu un sanctuaire célèbre. En fait, trois hypothèses s'offrent à nous. Isis Pharia possède un temple à Alexandrie, sur l'île de Pharos ou ailleurs: cela reste à démontrer. Isis possède un temple sur l'île de Pharos, qui pourrait lui valoir le qualificatif de Pharia: c'est possible, mais aucun document littéraire ou archéologique décisif ne permet encore de l'affirmer. Enfin Isis possède un temple à Alexandrie, et c'est son assimilation littéraire à Io qui lui vaut d'être qualifiée par métonymie de Pharia (= alexandrine ou égyptienne): cela ressort nettement de la documentation; aller plus loin serait extrapoler.

Cependant, la présence de cette épiclèse sur plusieurs documents épigraphiques vient apporter des éléments importants à verser au dossier.

Dans le poème funéraire de l'épicurien Agricola, de Tivoli, celui-ci évoque la mémoire de son épouse Primitiva Flavia, adoratrice de la déesse du Phare (cultrix deae Phariae), i. e. Isis. L'inscription peut être datée du milieu du III ${ }^{\mathrm{e}} \mathrm{s}$. après J.-C. L'expression doit ici encore être comprise comme une périphrase stylistique désignant Isis en tant que déesse égyptienne, plutôt que comme l'indice de l'existence, à Rome, d'un sanctuaire d'Isis Pharia auquel la défunte aurait été attachée ${ }^{(21)}$.

Dans deux proscynèmes peints de Haute-Égypte et de Nubie, datables de la fin $\mathrm{du}_{\mathrm{II}}^{\mathrm{e}} \mathrm{s}$. après J.-C., les pèlerins précisent qu'ils ont été élevés auprès d'Isis Pharia ${ }^{22}$ ). Là encore, il est permis d'hésiter. Doit-on comprendre qu'ils ont grandi à proximité d'un sanctuaire d'Isis Pharia - Isis du Pharos - ou bien simplement qu'ils affichent ainsi leur origine

(19) Apol. 16. (197 après J.-C.). Il y est question cette fois de Pallas Attica et de Ceres Pharia.

(20) Carmen IV, 99 = Anth. Lat. I, 1 pp. 13sqq (fin IV ${ }^{\mathrm{e}}$-début $\mathrm{V}^{\mathrm{e}} \mathrm{s}$.).

(21) CIL VI 17985a (Bücheler, CLE 856; SIRIS [= L. VIDMAN, Sylloge Inscriptionum Religionis Isiacae et Sarapiacae $=R G V V .28($ Berlin, 1969)] 451; M. MALAISE, Inventaire préliminaire des documents égyptiens découverts en Italie = EPRO. 21 (Leyde, 1972), pp. 127-128 Roma 51; RICIS [= L. BRICAULT, Recueil des Inscriptions concernant les Cultes Isiaques (à paraitre)] 501/0177; cf. E. SABBADINI, «Culto isiaco ed epicurismo nell'epitaffio di Flavio Agricola tiburtino", AST 48 (1975), pp. 71-72 [non vidi]). Rome. L'inscription, gravée sur une base cubique qui aurait été trouvée en 1626 sous la basilique SaintPierre, semble aujourd'hui perdue.

(22) 1) $C I G$ III 5119 (SB V 8542). Hiéra Sykaminos. Proscynème de Maximus peint sur l'épistyle du temple d'Isis. - 2) E. BERnAND, Les inscriptions grecques et latines de Philae, II. Haut et Bas-Empire (Le Caire, 1969), pp. 166-174, $\mathrm{n}^{\circ} 168$. Epigramme de Serenus lae, II. Haut et Bas-Empire (Le Caire, 1969), pp. 166-174, $\mathrm{n}^{\circ} 168$. Epig
peinte sur le mur ouest de la porte d'Hadrien (25 mars 191 après J.-C.). alexandrine? Dans ce dernier cas, Isis Pharia désignerait de nouveau l'Isis d'Alexandrie, sans plus de précision. Une autre inscription, retrouvée sur un îlot du lac Maréotis, concerne la consécration d'une statue de marbre par un certain Dioscoros à la très grande déesse Isis (surnommée Pharia?) (23). La dédicace est malheureusement mutilée et les lectures proposées ne garantissent pas le sens à donner à l'épiclèse, si elle est bien à restituer dans ce texte.

Ce sont les autres documents épigraphiques qui vont nous permettre d'avancer. Une dédicace à Isis Pharia d'une statue d'Isis de Ménouthis ${ }^{(24)}$ pour le salut d'un empereur Antonin $\left({ }^{25}\right)$, provient du Portus Ostiae $\left.{ }^{26}\right)$. Les rapports entre le Port d'Ostie et Alexandrie sont très étroits depuis la mise en service de ce nouveau havre par Trajan. La présence des Alexandrins y est forte, comme en témoignent les nombreuses inscriptions en grec qui furent retrouvées sur le site, dont une grande partie reste encore à fouiller $\left.{ }^{27}\right)$. La présence d'Isis n'est pas faite pour surprendre dans ce contexte, non plus que celle de Sarapis $\left({ }^{28}\right)$. Il est vraisemblable que c'est au sein d'un édifice religieux isiaque que fut placée la statue d'Isis de Ménouthis, dont on ne peut hélas déterminer quelles étaient les caractéristiques essentielles, tant iconographiques que proprement fonctionnelles. Sans doute quelque trait stylistique devait la distinguer d'Isis Pharia, sa voisine géographique. Toutefois, le document n'est

(23) AFP 2 (1903), p. 566, nº 123; E. BRECCIA, Iscrizioni (Alexandrie,1911), p. 67 , $\mathrm{n}^{\circ} 103$.

(24) Isis avait un temple à Ménouthis (cf. ÉPIPHANE DE SALAMINE, De fide 12,1-12,4); sur cette ville, voir A. BERNAND, Confins libyques (Le Caire, 1970), pp. 296-299; cf. également RICIS 503/1212 pour une seconde dédicace d'une statue d'Isis de Ménouthis, toulement RICIS 503/1212
jours du Portus Ostiae.

(25) On a longtemps hésité, et les avis divergent toujours, sur la personne de cet Empereur: Antonin le Pieux, Commode ou Caracalla?

(26) J. GRUTER, Inscriptiones (1602), pl. $85 \mathrm{n}^{\circ} 1$ (inscription trouvée dans une maison de Rome et vendue en 1843 au Musée de Parme); J.-A. Letronne, Recueil des inscription s grecques et latines de l'Égypte. I (Paris, 1842), pp. 434-437, $\mathrm{n}^{\circ} \mathrm{XLV}$, la considéra, d'aprè son contenu, comme originaire d'Alexandrie ou de ses environs, information reprise ensuite par Franz (CIG 4683b; il mentione l'origine romane de la piere das les Addenda, p.

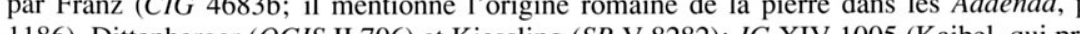
1186), Dittent vilégie l'origine romaine) (SIRIS 403); L. MORETTI, "Sulle iscrizioni greche di Porto», RPAA 1964, pp. 198-201, ${ }^{\circ}$ 4, fig. 3, attribue ce texte au Portus Ostiae (M. MAlaISE, Inventaire, p. 93 Portus Ostiae 14); A. Bernand, Confins libyques, pp. 296-299 (ph); 1 Porto 9 (ph); RICIS 503/1204.

(27) Elles ont fait l'objet d'un beau corpus dû à G. SACCO, Iscrizioni greche d'Italia Porto (Rome, 1984) [= I. Porto $]$.

(28) Sur les cultes isiaques à Ostie, voir M. Floriani SquarCiaPINO, I Culti orientali ad Ostia $=$ EPRO. 3 (Leyde, 1962), pp. 19-36. 
pas davantage explicite que les précédents sur la nature d'Isis Pharia, qui peut encore n'être que l'Isis d'Alexandrie ${ }^{29}{ }^{29}$.

Les mêmes conclusions peuvent être tirées de l'examen d'une inscription latine découverte en Crète à Phoenix, l'actuelle Loutro, et datée du règne de Trajan $\left({ }^{30}\right)$. Il s'agit d'une dédicace à Jupiter Soleil Très bon Très grand Sarapis et à tous les dieux ainsi qu'à l'empereur Trajan, effectuée par Epictetus, affranchi impérial, commissionnaire (tabellarius) $\left.{ }^{(31}\right)$, par l'intermédiaire de Dionysios, fils de Sostratos, Alexandrin, commandant d'un navire aux armes d'Isis Pharia appartenant à Titus Claudius Théon. Ce dernier doit être lui aussi un Alexandrin, comme l'indique le parasème de son bateau. On peut supposer qu'il fit une escale à Phoenix, sur la côte méridionale de la Crète, sur le chemin qui le menait d'Alexandrie en Italie. Les navires au nom et aux armes d'Isis sont assez nombreux, et ce n'est pas ici le lieu d'insister sur ce point ${ }^{\left({ }^{32}\right)}$. La nature de sa cargaison reste cependant indéterminée.

Une dernière inscription, fort courte, mérite attention. Il s'agit du nom

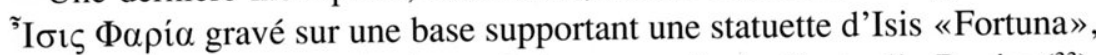
dont la provenance déclarée est Balanea, en Syrie, l'actuelle Banjas $\left({ }^{33}\right)$. Pour A. de Ridder, qui la publia le premier, «la base n'est pas sûrement celle de la figurine» $\left.{ }^{34}\right)$; dans l'impossibilité où nous sommes d'avoir pu examiner cet objet ${ }^{\left({ }^{3}\right)}$ ), il n'est possible ni d'infirmer, ni de confirmer avec certitude la justesse de cette remarque, que l'on peut toutefois soupçonner avoir été suscitée par l'apparente inadéquation entre le type représenté par la statuette et l'épiclèse figurant sur la base. Cependant, il serait intéressant de savoir dans quelle mesure une représentation d'Isis portant avec le

(29) Je ne suis guère convaincu par la démonstration de L. CASTigLIONE, «Isis Pharia Remarque sur la statuette de Budapest», Bulletin du Musée Hongrois des Beaux-Arts 34-35 (1970), pp. 37-55, figg. 24-39, pour qui à Sarapis / Osiris de Canope aurait correspondu une Isis Pharia alexandrine, puisque ce serait d'Alexandrie qu'Isis s'embarquait sur la mer à la recherche de son époux, depuis un sanctuaire que ce savant présume avoir existé sur l'île de Pharos.

(30) CIL III 3 (ILS 4395); I. Cret. II XX, 7 (p. 228) (SIRIS 171; RICIS 203/0701). 102 114 après J.-C. d'après la titulature impériale.

(31) Sur le tabellarius présent sur les navires alexandrins, cf. SÉNÈQuE, Epist. 77,1.

(32) Voir les références réunies dans le commentaire de l'inscription RICIS 115/0401.

(33) A. DE RIDDER, Catalogue de la collection de Clercq III. Les bronzes (Paris, 1905), p. 225, $\mathrm{n}^{\circ} 321$ et pl. L, 3 (IGLS IV, 1955, 1309; SIRIS 358; cf. Ph. BRUNEAU, op. cit. [n. 3] 1974, p. 349 et fig. $10 ;$ RICIS $402 / 0501$ ).

(34) Ibid., p. 225

(35) Il n'est pas localisable au Musée du Louvre, comme $\mathrm{Ph}$. Bruneau le notait déjà en 1974. bras gauche la cornucopia et tenant le gouvernail de la dextre pourrait symboliser, au sens figuré, la bonne fortune, et ne pas être également comprise, au sens propre, comme une déesse marine (le gouvernail) et propice (la cornucopia) ${ }^{(36}$ ). La réunion de ces deux fonctions, déesse de la mer ou protectrice de la navigation, mais aussi déesse propice qui apporte une heureuse vie, évoquerait assez bien, me semble-t-il, une situation très particulière: l'annone apportée chaque année à Rome par la flotte frumentaire d'Alexandrie $\left.{ }^{(37}\right)$.

S'il en était bien ainsi, l'Isis Pharia, c'est-à-dire l'Isis d'Égypte ou d'Alexandrie des poètes, serait devenue, par un glissement de sens aisément compréhensible, dans lequel le jeu de mot subtil entre $\varphi \tilde{a} \rho$ s $_{\text {(la }}$ voile) et $\Phi \alpha ́$ ó (le phare) soupçonné par Ph. Bruneau a peut-être joué un rôle ${ }^{\left({ }^{8}\right)}$ ), la déesse protectrice des convois de céréales assurant le ravitaillement de Rome. Quelques documents vont nous permettre d'étayer cette hypothèse.

Dans les Ménologes rustiques (Colotianum et Vallense), pour le mois d'avril, sont mentionnées deux fêtes qui doivent pouvoir être rapportées au sujet qui nous occupe: le Sacrum Phariae ${ }^{(39}$ ) et, probablement en même temps (item), les Serapia. Cette dernière fête se retrouve dans le calendrier de 354 , dit «de Philocalus», et se place précisément le 25 avril $\left({ }^{40}\right)$. R. Merkelbach avait déjà remarqué que cette date correspondait au 29 Pharmouthi du calendrier en usage à Alexandrie, soit la veille du $1^{\text {er }}$ Pachôn, date à laquelle on célébrait en Égypte, justement, une importante fête des moissons $\left({ }^{41}\right.$ ). La relation entre ces deux fêtes du 25 avril (Sacrum Phariae

(36) Cette idée avait déjà été émise par M. MaLAISE, Les conditions de pénétration et de diffusion des cultes égyptiens en Italie $=$ EPRO. 22 (Leyde, 1972), p. 181.

(37) On notera qu'en l'an 15 de Trajan, une émission monétaire d'Alexandrie montre Isis a la vole entre les deux Dioscures $\left(B M C \mathrm{n}^{0} 451\right)$, eux aussi protecteurs bien connus de

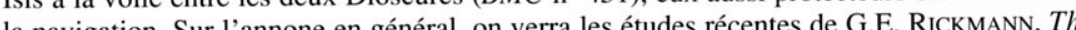
Corn Supply of Ancient Rome (Oxford, 1980); A.J.B. SiRKS, Food for Rome. The Legal Structure of the Transportation and Processing of Supplies for the Imperial Distributions in Rome and Constantinople (Amsterdam, 1991), ainsi que les contributions au volume Le ravitaillement en blé de Rome, E. GABBA (éd.) (Naples - Rome 1994), qui fournissent toute l'abondante documentation antérieure.

(38) Ph. BRUNEAU, op. cit. [n. 3] (1961), p. 444; l'idée fut reprise par P. NASTER, «Isis Pharia sur les monnaies impériales d'Alexandrie», BSFN 23 (février 1968), pp. 238-240. (39) CIL II 1, p. 358; CIL VI 2305-2306; CIL I' ${ }^{2}$ pp. 280-281; RICIS 501/0219.

(40) CIL I I , p. 262; RICIS 501/0221; voir H. STERN, Le Calendrier de $354=$ Biblio(40) CIL $\mathrm{I}^{2}$, p. 262; RICIS 501/0221; voir
thèque archéologique et historique. 55 (1953).

(41) R. MERKELBACH, Isisfeste in griechisch-römischer Zeit. Daten und Riten (Meisenheim am Glan, 1963), p. 43; il n'est pas question du sacrum Phariae dans cet ouvrage. Voir également le rapprochement fait par M. MALAISE, op. cit. [n. 36], p. 229 et, plus 
et Serapia) et l'annone semble confirmée par un important document archéologique $\left({ }^{42}\right)$. Une pièce, partie intégrante du Caseggiato dei Molini, à Ostie (Reg. I, Ins. III, 1), lequel est désormais identifié comme une bouOstie (Reg. I, Ins. II, 1), lequel nom de «sacellum de Silvanus» $\left({ }^{43}\right)$. Il s'agit d'un laraire privé dont la décoration peut être datée entre 210 et 235 après J.-C. environ. Les murs latéraux présentent un ensemble de peintures extrêmement intéressantes. Sur l'un des murs, on reconnaît trois groupes de personnages: un premier avec un cheval et les Dioscures, puis un deuxième avec Auguste, Harpocrate tenant la cornucopia, Isis brandissant le sistre, Fortuna tenant la cornucopia et un gouvernail, Annona tenant la tessera frumentaria et un gouvernail, un Genius (celui de l'Empereur?) tenant une cornucopia, et Alexandre le Grand, enfin un troisième où l'on devine au moins un personnage et Silvanus. Sur le mur opposé, on retrouve les mêmes personnages, à la différence qu'Isis n'est plus représentée, remplacée par une figure mâle tenant une patère dans la dextre ${ }^{(44)}$. L'ensemble est à rapporter au règne de Caracalla, d'autant qu'un graffito marque le passage en ces lieux de deux personnes (Marius et Anna) le 25 avril 215 après J.-C., précisément le jour du Sacrum Phariae et des Serapia $\left({ }^{45}\right)$. J.Th. Bakker rappelle d'ailleurs $\left({ }^{46}\right)$, à juste titre, qu'à cette même date, au $\mathrm{I}^{\mathrm{er}}$ s. après J.-C., on célébrait à Ostie la fête des Robigalia, en l'honneur de Robigus, une divinité qui protégeait les céréales de la rouille $\left(^{47}\right)$. Il est tentant de considérer que les Serapia ont, au $\mathrm{III}^{\mathrm{e}}$ s., remplacé cette antique fête. Cet ensemble pictural associe clairement les divinités isiaques à l'arrivée de l'annone à Ostie et réaffirme le lien étroit existant alors entre culte impérial (avec Caracalla comme nouvel Auguste et

généralement, Fr. PERPILLOU-Thomas, Fêtes d'Égypte ptolémaïque et romaine d'après la documentation papyrologique grecque = Studia Hellenistica. 31 (1993).

(42) Je tiens à remercier tout particulièrement mon ami et collègue Miguel John Versluys d'avoir attiré mon attention sur ce document.

(43) Cf. J.Th. BAKKER, Living and Working with the Gods. Studies of Evidence for Prirate Religion and its Material Environment in Ostia (100 BC - $500 \mathrm{AD})$ (Amsterdam, 1994), pp. 135-166.

(44) J.Th. BAKKER, op. cit., p. 152 suggère un Genius; je songerais plus volontiers à Sarapis.

(45) On rapprochera de cette date une émission monétaire de Caracalla dont le type de revers n'est attesté précisément que pour cette année 215. Il s'agit d'une frappe d'aurei (RIC IV, 1, n $\mathrm{n}^{\circ} 257 \mathrm{a}-\mathrm{b}, \mathrm{p} .249$ ) et de sesterces (RIC IV, $1, \mathrm{n}^{\circ} 544$, p. 303). Sur le revers, l'Empereur est debout à gauche, en militaire, tenant une lance et posant le pied droit sur un crocodile; Isis, face à lui, debout, avançant à dr., tient le sistre de la main gauche et lui présente de la main droite 2 épis de blé.

(46) Ibid., p. 166

(47) CIL XIV S 4547 nouvel Alexandre) et culte isiaque; il n'est donc pas étonnant de retrouver Isis, Harpocrate, et peut-être Sarapis dans la chapelle attenante à une boulangerie d'Ostie. On ignore toutefois qui commanda ces peintures: un simple boulanger, le corpus pistorum, ou bien une guilde religieuse? On notera que, parmi la quinzaine de statuettes retrouvées in situ, figurent deux Sarapis en bronze $\left({ }^{48}\right)$. Rappelons enfin que Tertullien, qui écrit son Apologétique en 197 après J.-C., évoquant Isis, lui donne le nom de Ceres Pharia $\left.{ }^{(49}\right)$, associant clairement l'épiclèse Pharia à la fertilité agraire.

Dès lors, si l'on admet qu'à partir du $\mathrm{II}^{\mathrm{e}}$ s. après J.-C., Isis Pharia est devenue la protectrice de l'annone, dont on sait qu'à $50 \%$ elle provient d'Égypte, et par là même que la déesse garantit, avec son parèdre Sarapis, le ravitaillement de Rome et le bien-être des habitants de l'Urbs, d'autres éléments vont s'expliquer plus aisément.

On a beaucoup discuté, à la suite d'A. Alföldi $\left({ }^{50}\right)$, du sens à donner à une série de médaillons émise en $190\left(^{(51)}\right.$ (fig. 1). Au revers de ceux-ci, on voit Commode offrant un sacrifice (sacrum) au pied d'un phare à quatre étages, à l'entrée d'un port qui doit être celui d'Ostie ( $\left.{ }^{52}\right)$; vers celui-ci se

48) J.Th. BAKKER, op. cit., chap. 5B

(49) Apol. 16.

(50) A. ALFÖLDI, A Festival of Isis in Rom under the Christian Emperors of the Ivth Century = Dissertationes Pannonicae. Ser. II, 7 (Leipzig, 1937), pp. 48-49, et ID., «Die alexandrinischen Götter und die vota publica am Jahresbeginn», JAC 8/9 (1965-1966), pp. 54-57.

(51) H. COHEN, Description historique des Monnaies frappées sous l'Empire Romain, Paris, no 993; F. GNECCHI, I Medaglioni romani. II (1912), p. 71, no 174 et pl. 89, 6-8 (pour le revers); W. SZAIVERT et al., Moneta Imperii Romani, $\mathrm{n}^{\mathrm{os}} 1139-3 / 46$; H. KütHMANn et al. Bauten Roms auf Münzen und Medaillen. Ausstellungskatalog Staatliche Münzsammlung München 1973, $\mathrm{n}^{\circ} 152$. L'exemplaire présenté ici, d'une qualité exceptionnelle, a été récemment mis en vente à Munich; cf. Auktion Lanz 94, Sammlung Leo Benz, 22. November $1999, \mathrm{n}^{\circ} 674$ et pl. couleurs 15.

(52) Pour plusieurs auteurs, dont $\mathrm{Ch}$. PICARD, «Sur quelques représentations nouvelles (52) Pon plexandrie et sur l'origine alexandrine des paysages portuaires $B C$ (1952), pp. 61-95, le phare du pot d'Ostic serat d'alleurs une imitation de celui d'Alexan(1952), pp. 61-95, le phare du port d'Ostie serait d'aillurs une inte drie, comme le montrerait la mosaique du Palais des Conservateurs trouvee sur le Quirinal (pp. 88-89 et fig. 12) ou encore le relief Torlonia provenant d'Ostie (p. 88 et fig. 13) et montrant le Portus. La scène portuaire du couvercle du sarcophage de Iulius Filocyrius (pp. 91-92, fig. 14), datant de la seconde moitié du $\mathrm{III}^{\mathrm{e}} \mathrm{s}$. après J.-C., trouvé à Ostie, symbolise également Alexandrie par bien des détails. On pourrait ajouter que le temple que l'on distingue à l'extrême gauche de la scène pourrait bien être l'Iseum. J. LECLANT, IBIS. III (1985) $n^{\circ} 1087$, fisait en outre remarquer que le défunt figuré dans un clipeus est coiffé de (1985), no (a VON GONZENBACH, Untersuchungen zu den Knabenweihen im Isishuth dor romischen Kaiserzeit (Bonn, 1957), pp. 147-148, $\mathrm{n}^{\circ} \mathrm{K} 16 \mathrm{et}$ pl. 15; sur ce document, c. L. Musso, BCAR 93 (1989-1990), pp. 294-299 (ph.) (AE 1991, 312), R. MERKELBACH, Zets Sarapis - Isis regina (Stuttgart - Leipzig, 1995), pl. 126; RICIS 503/1122. Pour le symbolisme des scènes 
dirigent cinq navires, deux grandes galères toutes voiles dehors et trois embarcations plus petites, l'une à quatre rameurs, les deux autres avec un seul rameur, qui doivent leur servir de pilote. À la poupe de la plus grande galère siège Sarapis, reconnaissable à son calathos, tandis qu'un vexillum se dresse à la proue. Aux pieds de l'Empereur, on reconnaît un taureau, que l'on pourrait comprendre comme une victime sacrificielle, d'autant qu'une patère est figurée devant la bête. Comme l'atteste la légende de revers, cette frappe est à mettre en relation avec des Votis felicibus qu'A. Alföldi avait songé tout d'abord à identifier aux Vota publica du Nouvel An, voire avec le Navigium Isidis du 5 Mars. Ce rapprochement, assez subtil, entre les Vota publica du 3 Janvier et la fête de l'ouverture de la navigation a été critiqué, à juste titre, par plusieurs savants $\left({ }^{53}\right)$. J. Beaujeu considérait quant à lui que ces Vota felicia étaient plutôt à mettre en relation avec la Felicitas de la propagande impériale, intimement liée à l'annone. En effet, en 189, une très violente famine avait secoué Rome, provoquant des émeutes au cours desquelles Cléandre, le favori de l'Empereur, avait péri ( $\left.{ }^{54}\right)$. L'approvisionnement en blé de Rome, crucial, ainsi placé officiellement au début de 190 sous la protection de Sarapis et d'Isis, devait permettre d'éviter qu'une telle tragédie se reproduise. On peut alors songer que cette émission eut lieu à l'occasion des fêtes du Sacrum Phariae et des Serapia du 25 avril. C'est en ce sens que Sarapis peut apparaitre comme conservator Augusti sur certaines monnaies de Commode frappées en 191 et $192{ }^{(55)}$. Pour 192, le type de revers

nilotiques et portuaires en général, on verra les remarques de J. LECLANT, «Un relief pannonien d'inspiration égyptisante», $R A$ 1950/II, pp. 147-149. Cette influence alexandrine dans les paysages portuaires était, semble-t-il, déjà sensible pour Pouzzoles, qui précéda Ostie comme destination du commerce alexandrin en Italie; cf. Ch. PICARD, «Pouzzoles et le paysage portuaire», Latomus 18 (1959), pp. 23-51, XIV pll.

(53) Voir, entre autres, M. MESLIN, La fête des kalendes de janvier dans l'Empire romain (Paris, 1970), pp. 63-64, et M. MALAISE, op. cit. [n. 36], pp. 220-221. Noton d'ailleurs que, dans le cas présent, les navires entrent au port, ce qui se comprendrait mal 'il s'agissait d'une cérémonie d'ouverture de la navigation.

(54) J. BEAUJEU, La religion romaine à l'apogée de l'Empire. I. La politique religieuse des Antonins, 96-192 (Paris, 1955), pp. 379-381. Cet auteur songe même à identifier, p. 381, n. 4, ces vœux aux Vota decennalia qui auraient dû être célébrés en 190.

(55) RIC III $\mathrm{n}^{\circ} 601$, p. 434, avec pour légende de revers Serapidi Conserv(atori) Aug(ustus) Co(n)sul VI P(ater) P(atriae) S(enatus) C(onsultum), ainsi que les $\mathrm{n}^{\text {os }} 605$ et 607 , p. 435 , pour l'année 191. RIC III n ${ }^{\circ} 246$, p. 394, qui présente au revers Commode debout à gauche, couronné par la Victoire, les mains tendues au-dessus d'un autel, Sarapis portant le calathos et Isis tenant le sistre debout à droite, de même que le $\mathrm{n}^{\circ} 261$, p. 397, avec pour légende de revers Serapidi Conserv(atori) Augustus, et les n ${ }^{\circ s} 614 \mathrm{a}-\mathrm{b}$, p. 436 , 621, p. 437,628 , p. 437 et 630 , p. 438 pour l'année 192. employé, avec Commode sacrifiant devant Isis et Sarapis, doit pouvoir être rapporté à cette même cérémonie du 25 avril $\left({ }^{(56)}\right.$.
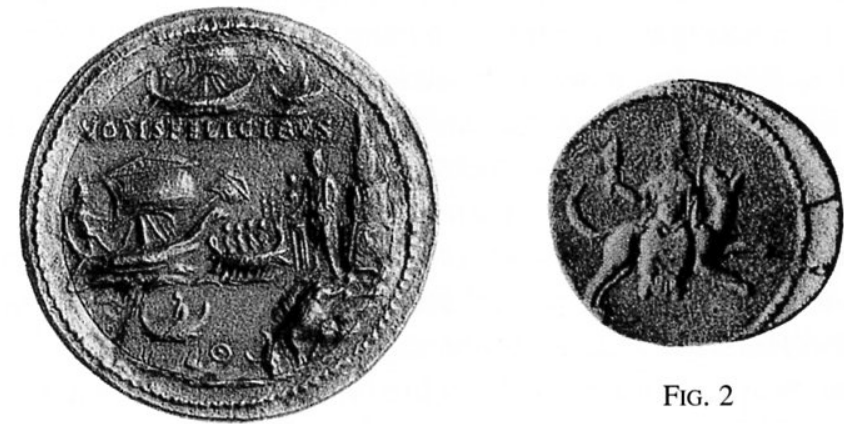

FIG. 2

FIG. 1

Nombreuses sont les dédicaces de cette époque provenant du Portus Ostiae et mettant en relation Isis, Sarapis et la flotte frumentaire d'Alexandrie. Citons par exemple une dédicace de 180-192 après J.-C., provenant probablement du Serapeum de Porto, déposée par les nauclères de la flotte marchande alexandrine, pour le salut et la permanence de l'empereur Commode $\left.{ }^{57}\right)$; la consécration, en 201 après J.-C., d'une statue d'Adrastée avec tout le décor qui l'entoure par Gaius Valerius Serenus, néocore du grand Sarapis, le curateur de toute la flotte d'Alexandrie, pour le salut, le retour et la permanence perpétuelle des empereurs Sévère, Antonin et Iulia Auguste, ainsi que pour tous ceux de leur maison, et pour l'heureuse navigation de toute leur flotte, Claudius Iulianus étant préfet de l'annone $\left({ }^{58}\right)$; ou encore la dédicace au dieu (i. e. Sarapis), par un nau-

(56) C'est peut-être à la commémoration de cette même cérémonie qu'il faut rapporter la monnaie de Philippopolis de Thrace [voir l'Annexe ci-après et fig. 5] datée du règne de Commode. L'image d'Isis à la voile dans cette ville très éloignée des côtes s'explique difficilement autrement.

(57) IG XIV 918; I. Porto 2 (ph.); M. BuONOCORE, Le iscrizioni latine e greche = Musei della Biblioteca Apostolica Vaticana. Inventari e Studi. 2 (Città del Vaticano, 1987), no 9 , pp. 37-39 et pl. VII, fig. 14; RICIS 503/1217. Le nom de Commode, après avoir été érasé, fut regravé, peut-être sous Sévère Alexandre. S'il est tentant de dater ce texte de la fin du règne, rien ne peut l'assurer.

(58) IG XIV 917 (IGRR I 380; SIRIS 552; M. MALAISE, Inventaire, pp. 91-92 Portus Ostiae 8 ; I. Porto 3 (ph); RICIS 503/1207. Ce personnage important a dédié aussi les inscriptions RICIS 503/1208-10. 
clère, d'une statue, pour le salut des empereurs (les Sévères sans doute) et de la splendide flotte $\left({ }^{59}\right)$

Un siècle plus tard, les séries bien connues, grâce aux travaux d'A. Alföldi, des monnaies à thèmes isiaques frappées à l'occasion des Vota publica, vont souligner davantage encore ce lien qui unit Sarapis, Isis Pharia et la flotte de l'annone. Dans les séries sur lesquelles apparaît le buste de l'Empereur, c'est-à-dire entre le règne de Dioclétien et celui de Gratien (379 après J.-C. précisément pour A. Alföldi), la légende du droit identifie le princeps. Sur les émissions anonymes qui vont suivre pendant plus de quinze ans, deux légendes vont accompagner le type du droit: Deo Sarapidi s'il s'agit de Sarapis seul ou du couple Sarapis / Isis, Isis Faria s'il s'agit d'Isis seule. Les multiples types isiaques ornant les revers de ces monnaies, qu'il s'agisse d'Isis à la voile, d'Anubis tenant la palme et le caducée, d'Isis chevauchant Sothis, d'Isis allaitant Harpocrate se rattachent tous à la thématique des vœux du Nouvel An (santé, paix, nourriture, prospérité, naissance), ainsi placés sous les auspices du dieu Sarapis et d'Isis Pharia. Il est d'ailleurs significatif que ces pièces frappées à Rome n'aient pas disparu après 331, lorsque la décision fut prise de réserver le blé d'Égypte aux besoins de Constantinople $\left.{ }^{60}\right)$. Le patronage d'Isis s'étendait donc aux autres flottes annonaires, dont celle d'Afrique, devenue la plus importante. Dans ce contexte, la décision prise officiellement, en théorie, au début de 376 après J.-C. par Valens, Gratien et Valentinien II de rendre à la déesse Isis son temple (du Portus Ostiae) et son portique n'est pas pour surprendre. C'est le clarissime Sempronius Faustus, préfet de l'annone, directement placé sous les ordres du préfet de la Ville (praefectus Urbi) - que l'on peut davantage soupçonner être à l'origine de cette décision - , alors en charge des opera publica à Ostie et au Portus, qui en assura l'éxécution $\left({ }^{61}\right)$. Ce document illustre à merveille le bras de fer qui opposa au $\mathrm{IV}^{\mathrm{e}} \mathrm{s}$. après J.-C. l'aristocratie païenne de Rome et le pouvoir impérial chrétien de Milan ou de Constantinople ( $\left.{ }^{62}\right)$.

(59) IG XIV 923 (IGRR I 383; I. Porto 20; RICIS 503/1215).

(60) Cf. A. Piganiol, L'Empire chrétien (Paris, 1947), pp. 48-51.

(61) SIRIS 562; A. CHASTAGNOL, «La restauration du temple d'Isis au Portus Romae sous le règne de Gratien", dans Hommages à M. Renard. II = Coll. Latomus. 102 (Bruxelles, 1969), pp. 135-144 (AE 1968, 86); L. VIDMAN, «Ein neuer «praefectus annonae» von Ostia», dans Studi in onore di E. Volterra. I (1971), pp. 207-211 (AE 1971, 67) (cf. L. VIDMAN, Isis und Sarapis bei den Griechen und Römern. Epigraphische Studien zur Verbreitung und zu den Trägern des ägyptischen Kultes $=$ RGVV. 29 [Berlin, 1970], pp. 161-162; M. MALAISE, Inventaire, pp. 93-94 Portus Ostiae 16 et ID., op. cit. [n. 36], pp. 450-451); RICIS 503/1223.

(62) L'étude magistrale d'A. Alföldi, op. cit. [n. 50], si elle doit être nuancée sur certains détails (cf. supra n. 53) et complétée par les documents nouveaux parus depuis 1937,
Il est temps de revenir, pour conclure, à Faustine et aux titres de Sôsistolos et de Pharia que le nouveau document d'Alexandrie lui attribue. Il semble bien que l'épouse de Marc Aurèle se soit posée de la sorte en protectrice de la flotte frumentaire d'Alexandrie, garante du bon acheminement du blé d'Égypte, à l'instar d'Isis à laquelle elle paraît s'être identifiée ${ }^{(63)}$. De très nombreuses monnaies émises au nom de l'Impératrice affichent des thèmes isiaques, dépassant en nombre celles de son époux et de son père ${ }^{(64)}$. Pour le seul atelier d'Alexandrie, on connaît au moins quatre séries d'émissions (drachmes et tétradrachmes), au revers desquelles on retrouve Isis à la voile $\left({ }^{65}\right)$. Pour Rome, il n'est que de mentionner le médaillon fameux qui présente au droit le portrait de Faustine, et au revers une Isis à la voile sur un navire, devant le Phare d'Alexandrie (fig. 3). Sans doute ce médaillon et la dédicace alexandrine à l'origine de cette enquête renvoient-ils à une même réalité $\left({ }^{66}\right)$.

\section{AnNeXe. La diffusion des monnaies du type d'Isis à la voile.}

Dans les publications numismatiques, les monnaies figurant Isis à la voile sont qualifiées systématiquement d'Isis Pharia, qu'elles proviennent

reste fondamentale. Nous reviendrons sur ce sujet dans un chapitre de la Sylloge Nummorum Religionis Isiacae et Sarapiacae (= SNRIS), en préparation en collaboration internationale.

(63) Je discuterai ailleurs de cette identification probable à Isis de l'épouse de Marc Aurèle; comparer déjà le médaillon publié par F. GNECCHI, I Medaglioni. III (1912), p. 34 $n^{\circ} 44$, qui présente au revers Isis-Sothis, une première dans le monnayage impérial (fig. 2); cf. également infra n. 66.

(64) Il n'est pas possible de citer ici toutes ces monnaies. La publication de la SNRIS, à l'horizon 2005, permettra de se faire une idée très précise de cette réalité.

(65) An 10 = 146/7: Drachme: Paris BnF CM nº inv. 1699bis (inédite); an 11 =147/8: Drachme: Frankfurt 691; Datt. 3289; Geissen 1943; SNG Milano 1333; Milne 1968; ANS

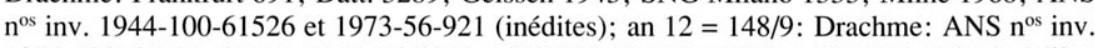
1944-100-61534, 61535, 1974-26-3844, 0000-999-25326, 25327 et 25328 (inédites); Milne 2009; $B M C$ 1338; Demetrio 2102; Paris $\mathrm{BnF} \mathrm{CM} \mathrm{n}{ }^{\circ}$ inv. 2570 (inédite); an $17=153 / 4$ : Tétradrachme: Frankfurt 696; ANS nos inv. 1944-100-61481, 61482 et 1974-26-3827 (inédites); Datt. 3250; Geissen 1970-1971; Milne 2213-2214; BMC 1326; Curtis 689-690; Demetrio 2122 .

(66) Médaillon de bronze: F. GNECCHI, I Medaglioni. III (1912), p. 34, $\mathrm{n}^{\circ} 43$ et pl. 151,4; notons encore les représentations suivantes d'Isis à la voile sur des monnaies de Faustine à Amastris: Fr. IMHOOF-BLUMER, Griechische Münzen, p. 89, pl. V, 13; RecGén I, 12 p. $181 \mathrm{n}^{\circ} 108$, pl. 20, 25; W. DREXLER, NZ 21 (1889), pp. 21-22, pl. 2, 14; et à Kyme: inédite, Paris BnF CM $215 \mathrm{n}^{\circ}$ inv. 2053; enfin, signalons dès à présent la série monétaire émise à Lystra en Lycaonie, au droit de laquelle Faustine est, semble-t-il, coiffée du basileion, tandis que le revers présente Isis assise, coiffée du basileion et tenant des épis de blé (BMC Lycaonia, Isauria and Cilicia, p. 10, $\mathrm{n}^{\mathrm{os}} 2-3$ ). 


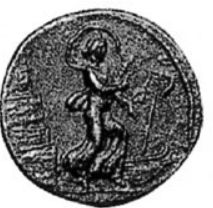

FIG. 3

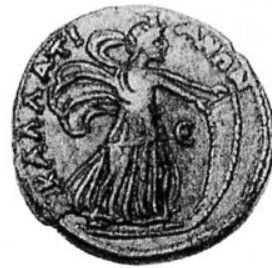

FIG. 4

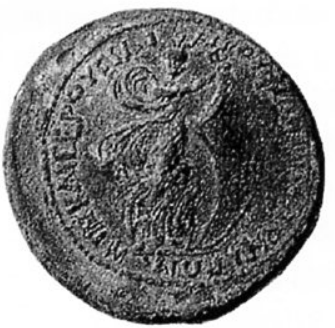

FIG. 5
d'Alexandrie ou d'ailleurs, que le phare s'y trouve représenté ou non. Cette terminologie, pour commode qu'elle soit, n'est guère pertinente, car simplificatrice et source de nombreuses confusions sur le sens à donner à ces émissions. Voir, par exemple, les études de S. HANDLER, «Architecture on the Roman Coins of Alexandria», AJA (1971), pp. 56-61, et Fr. Daumas $\dagger$ et B. MathiEu, "Le phare d'Alexandrie et ses dieux: un document inédit», $A W L s K$ 49, 1 (1987), pp. 43-54, avec la bibliographie antérieure. Des monnaies au type d'Isis à la voile sont signalées pour plusieurs cités, essentiellement portuaires, ce qui semble aller de soi (cf. cependant supra et $\mathrm{n}$. 56 pour une monnaie de Philippopolis de Thrace).

Byblos: dès l'époque séleucide avec Antiochos IV (175-164): E. BABElon, Catalogue des monnaies grecques de la Bibliothèque Nationale. Les rois de Syrie (Pais, 1890), p. 74, $\mathrm{n}^{\circ} 575=\mathrm{J}$. Rouvier, «Numismatique des villes de la Phénicie: Botrys, Gébal-Byblos, Césarée du Liban-Arca, Carné», JIAN 4 (1901), ${ }^{\circ}$ 650; ANS no inv. 1992-54-1588 (inédite); Antiochos VIII (12196): ANS $\mathrm{n}^{\text {os }}$ inv. 1944-100-77151, 77152 et 77153 et 1971-142-14 (inédites); $\mathrm{I}^{\mathrm{er}} \mathrm{s}$. avant J.-C.: RouviER $\mathrm{n}^{\circ} 670$; H. SEYRIG, $R N$ 1968, p. 18, $\mathrm{n}^{\circ}$ 340; SNG Schweiz II no 2257 (an $3=62 / 1$ ); Fr. IMHOOF-Blumer, Monnaies grecques, p. $443 \mathrm{n}^{\circ}$ 23, pl. J,3; ANS $\mathrm{n}^{\circ}$ inv. 1944-100-70835 (inédite); Claude (45/6): RouvIER $\mathrm{n}^{\text {os }}$ 680-681; BMC Phoenicia pp. 99-100 $\mathrm{n}^{\circ} 20$, pl. XII,10; RPC I 4528; (an $6=46 / 7$ ?) ANS $n^{\text {os }}$ inv. 1944-100-70856, 1944 100-70857, 1944-100-70859, 1944-100-70860 et 1948-19-2186 (inédites); Commode (180-192): BMC p. 104, $\mathrm{n}^{\text {os }} 22-24 ;$ SNG Cop. $\mathrm{n}^{\circ} 143$; ROUVIER $\mathrm{n}^{\circ}$ 686; ANS n ${ }^{\text {os }}$ inv. 1944-100-70861, 1944-100-70868 et 70869, 1948-192187 (inédites); Macrin (217-218): BMC p. 104, no 39; ROUVIER n ${ }^{\circ} 698$; ANS $\mathrm{n}^{\text {os }}$ inv. 1944-100-70890 et 1948-19-2190 (inédites); Diaduménien (217-218): ROUVIER $\mathrm{n}^{\circ} 702 ;$; $M C$ p. 104, nos 44-46, pl. XII,15; SNG Cop. $\mathrm{n}^{\circ}$ 144; SNG Schweiz II no2264; ANS $\mathrm{n}^{\text {os }}$ inv. 1944-100-70896 et 70897 (inédites); Élagabal (218-222): $B M C$ p. $107 \mathrm{n}^{\text {os }} 66-67$; voir également $\mathrm{Z}$. SAWAYA, «Cronos, Astarté: deux légendes phéniciennes inédites sur des

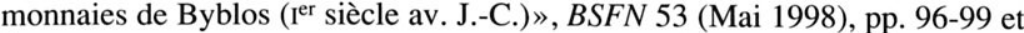
figg.

Alexandrie: entre 90 et 165 après J.-C.; le nombre considérable de monnaies de ce type, avec ou sans le phare, ne permet pas de les mentionner ici; je les commenterai dans une autre étude plus large consacrée à Isis marine. Notons simplement ici que le Phare apparaît pour la première fois sur des monnaies de ce type de l'an 15 de Trajan, c'est-à-dire en 111/112 après J.-C.

Amastris: Gordien III (238-244): ANS no inv. 1944-100-41524 (inédite); cf supra n. 66 pour une monnaie de Faustine la Jeune.

Anchialos: $A M N G$ II p. 218, ${ }^{\text {os }} 408-409$, pl. 5, 32.

Aspendos: Macrin (217-218): BMC Lycia p. 104, $\mathrm{n}^{\circ}$ 85; Diaduménien (217-218): Fr. IMHOOF-BLUMER, Kleinasiatische Münzen, p. 321, nº 45a, pl. 10, 31; SNG Aulock $\mathrm{n}^{\circ}$ 4589; Iulia Maesa: BMC Lycia p. LXXV.

Callatis: Septime Sévère (193-211): Classical Numismatic Group, Sale 49, nº 895 (inédite) (fig. 4)

Cleonae: (ép. imp.) B.V. HEAD, Historia Nummorum, pp. 441 et 621

Corinthe: plusieurs émissions de Trajan à Caracalla. Par ex., Lucius Verus (161169): ImHOOF et GARDNER, Numismatic Commentary on Pausanias p. 17 et pl. D, 64 (= JHS 6, 1885, p. 66); Plautille: Slg Weber II no 3811, pl. 140.

Éphèse: Gordien III (238-241): SNG Cop. $\mathrm{n}^{\circ}$ 549; Paris $\mathrm{n}^{\circ}$ inv. 962 (inédite); BMC Ionia $\mathrm{n}^{\circ}$ 422; W. DREXLER, NZ 21 (1889), pp. 87-88, pl. 2, 16.

Iasos: Lucius Verus (161-169): B.V. HEAD, Historia Nummorum, pp. 441 et 621.

Kyme: Type 1[pied arr. levé]: Antonin (138-161; monétaire Hiéronymos): Berlin $n^{\circ}$ inv. Kassel 1925 (inédite); Paris 212 A ( $n^{\circ}$ inv. 2035) (inédite); Valérien et Gallien (253-260; monétaire Elpidèphoros): SNG Aulock $\mathrm{n}^{\circ} 1647$; BMC Aeolis $\mathrm{n}^{\circ} 116$, pl. 23, 1; SNG Cop. $\mathrm{n}^{\circ}$ 128; Wien $\mathrm{n}^{\text {os }}$ inv. 16808, 30105 , 30491 et 136182 (inédites); Berlin $\mathrm{n}^{\circ}$ inv. 28732 (inédite); Type 2 [pied arr. posé] (monétaires Synèphorôn ou Asclèpiadès): Wien $\mathrm{n}^{\mathrm{o}}$ inv. 32897 (inédite); ANS ${ }^{\circ}$ inv. 1944-100-44148 (inédite); cf. supra $\mathrm{n}$. 66 pour une monnaie de Faustine la Jeune.

Magnésie du Méandre: Caracalla (198-217): BMC Ionia p. 166, nº 58, pl. 19,9; Sévère Alexandre (222-235): BMC Ionia p. 168, $\mathrm{n}^{\circ}$ 67A; Maxime (235238): BMC Ionia p. 170, $\mathrm{n}^{\circ} 81$.

Nicomédie: Antonin (138-161): ScholZ, NZ 1910, pp. 160-161; Marc Aurèle (161-180): RecGén II p. 527, nº 86, pl. 91, 8; Commode (180-192): FonTANA, RIN 1967, p. 44, n 4.

Périnthe: Sévère Alexandre (222-235): E. SCHÖNERT-GEISS, Die Münzprägung von Perinthos (Berlin, 1965), $\mathrm{n}^{\circ} 782$, pl. 48.

Philadelphie (Lydie): (Sévères): W. DREXLER, NZ 21 (1889), pp. 147-148.

Philippopolis (Thrace): Commode (177-192): G. Hirsch Nachf., Auktion 203, $\mathrm{n}^{\mathrm{o}} 601$ (inédite) (fig. 5).

Phocée: Maxime (235-238): BMC Ionia p. 226, no 156, pl. 23,18; SNG Cop. no 1069; W. DREXLER, NZ 21 (1889), pp. 104-106. 